\title{
Article \\ Effects of High-Intensity Electromagnetic Stimulation on Reducing Upper Limb Spasticity in Post-Stroke Patients
}

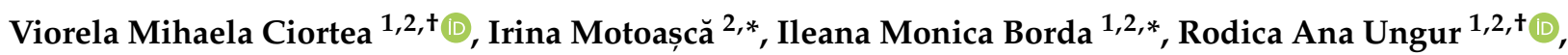 \\ Cosmina Ioana Bondor ${ }^{3}$, Mădălina Gabriela Iliescu ${ }^{4}+{ }^{+}$, Alina Deniza Ciubean ${ }^{1}$, Izabela Lazăr ${ }^{2}$, Eliza Bendea ${ }^{2}$ \\ and Laszlo Irsay $1,2,+$
}

check for

updates

Citation: Ciortea, V.M.; Motoașcă, I.; Borda, I.M.; Ungur, R.A.; Bondor, C.I.; Iliescu, M.G.; Ciubean, A.D.; Lazăr, I.; Bendea, E.; Irsay, L. Effects of High-Intensity Electromagnetic Stimulation on Reducing Upper Limb Spasticity in Post-Stroke Patients. Appl. Sci. 2022, 12, 2125. https://doi.org/10.3390/ app12042125

Academic Editor: Elena Amaricai

Received: 19 December 2021

Accepted: 16 February 2022

Published: 18 February 2022

Publisher's Note: MDPI stays neutral with regard to jurisdictional claims in published maps and institutional affiliations.

Copyright: (C) 2022 by the authors. Licensee MDPI, Basel, Switzerland. This article is an open access article distributed under the terms and conditions of the Creative Commons Attribution (CC BY) license (https:// creativecommons.org/licenses/by/ $4.0 /)$.
1 Department of Rehabilitation, "Iuliu Hatieganu" University of Medicine and Pharmacy, 40012 Cluj-Napoca, Romania; viorela.ciortea@yahoo.com (V.M.C.); ungurmed@yahoo.com (R.A.U.); alina.deniza.ciubean@gmail.com (A.D.C.); irsaylaszlo@gmail.com (L.I.)

2 Clinical Rehabilitation Hospital, 400347 Cluj-Napoca, Romania; izabelalazar@yahoo.com (I.L.); lizuk_me2@yahoo.com (E.B.)

3 Department of Medical Informatics and Biostatistics, "Iuliu Hatieganu" University of Medicine and Pharmacy, 40012 Cluj-Napoca, Romania; cosmina_ioana@yahoo.com

4 Department of Rehabilitation, Faculty of Medicine, “Ovidius" University of Constanta, 906100 Techirghiol, Romania; iliescumadalina@gmail.com

* Correspondence: motoascairina@gmail.com (I.M.); monicampop@yahoo.fr (I.M.B.); Tel.: +40-723-009-928 (I.M.B)

+ These authors contributed equally to this work.

\begin{abstract}
Super Inductive System (SIS) stimulation of spastic limbs by tissue-induced electromagnetic field may have the effect of reducing spasticity and improving functionality in patients with poststroke spasticity. The aim of the study was to evaluate two different protocols for the application of SIS on upper limb spasticity after stroke. We included 60 patients with post-stroke upper limb spasticity, who were randomized into two groups: the study group, with a 9 min application protocol ( $1 \mathrm{~min}$ for agonist muscles, $8 \mathrm{~min}$ for antagonistic muscles); and the control group, with an $8 \mathrm{~min}$ protocol applied only to the antagonistic muscles. The duration of therapy was 10 days, and the results were assessed using the Modified Ashworth Scale (MAS) and the Barthel Index. Both the MAS and the Barthel Index improved significantly after 10 days of treatment $(p<0.001)$, but 30 days after the completion of therapy, there was an attenuation of the effects in both study groups. The study group had a significantly higher percentage of patients with improved MAS after 10 days $(p=0.004)$ and within 30 days $(p<0.001)$ than the control group. An SIS protocol applied on both agonist and antagonist muscles has a more pronounced and longer lasting spasticity-reducing and improved functionality effect than its application on only antagonistic muscles.
\end{abstract}

Keywords: spasticity; stroke; high-intensity electromagnetic field stimulation; Super Inductive System

\section{Introduction}

Stroke is a serious health problem, being the world's second leading cause of death, with 5.5 million deaths annually, according to World Health Organization statistics [1]. It is also the leading cause of long-term disability in adult patients with neurological disorders [2]. Approximately $80 \%$ of strokes are ischemic, the most common disability being the motor deficit of the upper and lower limbs, and $50 \%$ of patients surviving post-stroke remain with a permanent disability [3]. This condition involves long-term rehabilitation treatments and, consequently, increased costs [4]. Recovery from ischemic stroke is conventionally considered to bring important benefits within the first 3 months, with a cessation of progress being observed 6 months after the stroke. However, there are recovery methods that have proven effective even after this period of time. Late functional recovery, most commonly between 3 months and 12 months, is reported to take place in 
one in four patients after an ischemic stroke, but no clear conclusion can be drawn about the consequences of late long-term rehabilitation in post-stroke patients [5].

Post-stroke patients may need both the support of their family/caregivers and recovery treatments for a shorter or longer period of time, or for the rest of their lives, in order to manage sequelae and/or to develop compensatory means. The management of these patients involves methods to combat spasticity, improve functionality and gait, manage swallowing impairments and depression, improve participation and patient motivation, and understanding the need for rehabilitation treatment in general and the concept of self-rehabilitation [1].

The action of the electromagnetic field causes changes in the cell membrane. It also acts upon the metabolism of blood electrolytes and cellular metabolic processes, its effects being dependent on the intensity and frequency applied.

Repetitive peripheral magnetic stimulation (rPMS) influences the opening of membrane channels with consequences on mitochondrial metabolism and mitotic activity in nuclear components. Previous studies have shown favourable effects on the proliferation and differentiation of osteocytes and mesenchymal stromal cells with implicit acceleration of the healing process, resulting in the reduction in rehabilitation period [6]. Moreover, regenerative effects on the peripheral nervous system of the pulsed electromagnetic field have also been demonstrated [7].

Repetitive peripheral magnetic stimulation is used in the recovery of patients with central and peripheral neurological disorders in order to improve motor function, with the possibility of penetrating deep into the tissues $[4,8]$.

High-intensity electromagnetic stimulation can improve the functionality of poststroke patients, the principle of action being based on the induction of an electromagnetic field that interacts with human body tissues, including myoarthrokinetic tissue, subsequently giving rise to electric currents to reduce spasticity. High-intensity electromagnetic stimulation (Super Inductive System) determines the depolarization of neuronal cells, generating therapeutic effects such as: analgesia, myostimulation, and trophic, resorptive, and elasticizing effects $[9,10]$.

Super Inductive System Therapy restores distorted muscle balance after stroke, both by relaxing the spastic muscles and by stimulating the weak muscles, as well as by the trophic and circulatory effects at the level of the area where the therapy was applied $[9,11]$.

Super Inductive System Therapy also has proven analgesic effects and improves the performance of daily activities in patients with acute and chronic musculoskeletal or neurological disorders. The analgesic effect of the Super Inductive System is based on the three neurophysiological theories of pain control, with frequency-dependent effects. Frequencies of 2-10 Hz stimulate the secretion of analgesic opioids by acting on the nerve endings; the frequencies of $60-100 \mathrm{~Hz}$ act according to the theory of gate control on the thick, myelinated nerve fibres with fast conduction speed; and frequencies between 120 and $140 \mathrm{~Hz}$ act according to the theory of the peripheral pattern, the coded information not being interpreted as pain [12].

Super Inductive System Therapy is a non-invasive, painless, well-tolerated method of treatment, without the nephrotoxic and hepatotoxic side effects of drug therapies; and can be used in the recovery of patients with various conditions associated with the basic pathology [12-14].

The aim of this study was to evaluate whether two different protocols of application of the high-intensity electromagnetic stimulation (Super Inductive System) as part of a physical therapy program can decrease spasticity and increase the muscle contraction force of the post-stroke spastic upper-limb.

\section{Materials and Methods}

\subsection{Patient Selection}

In the study, we enrolled 60 adult patients (over 18 years old) that were hospitalized for post-stroke rehabilitation therapy in our centre between 4 December 2019 and 30 May 2021. 
The inclusion criterion was the presence of upper-limb spasticity, irrespective of the elapsed time from the cerebral vascular accident. The exclusion criteria were the spasticity of causes other than stroke; the carrying of a cardiac pacemaker, defibrillator, neurostimulator implant, electronic implant or metallic implant; and some comorbidities which contraindicate electrotherapeutic treatment, including high-intensity electromagnetic stimulation: respiratory failure, coagulation diseases or anticoagulant therapy, cardiac disease, cancer, fever, or pregnancy.

\subsection{Treatment Protocol}

The 60 patients were assigned, by randomization, at the beginning of the therapy, to 2 treatment groups. In the study group, 29 patients received 10 therapy sessions of $9 \mathrm{~min}$ each, using the Super Inductive System. The applicator was placed first on the agonist (flexors of the forearm) muscles for $1 \mathrm{~min}$, for post-facilitatory inhibition and a decrease in spasticity, and afterwards on the antagonist (extensors of the forearm) muscles for $8 \mathrm{~min}$, in order to increase the muscle strength to obtain a higher blood perfusion and trophic improvement. The other 31 patients (control group) received 10 therapy sessions using the Super Inductive System, with a duration of $8 \mathrm{~min}$ for each session. The applicator was placed on the antagonist muscles (extensors of the forearm). Patients in both groups also received the standard kinesitherapy protocol for spasticity. No new treatment method was tested, as the Super Inductive System is approved for physical therapy by the Ministry of Health. The study was conducted according to the Declaration of Helsinki and was approved by the University Ethics Committee.

\subsection{Devices}

The device used in the study was the BTL-6000 Super Inductive System Elite, an electromagnetic wave therapy device with a maximum intensity of 2.5 Tesla and a frequency between 1 and $150 \mathrm{~Hz}$. The applicator of the device was positioned on the treated area using the non-contact technique, at a distance of 1-2 cm from the skin.

A sequence of 6 sections was applied in the study group, and 5 sections in the control group. The intensity was tested individually for every patient at the beginning of each therapy session and gradually increased up to the motor threshold, which represents 15-25\% of the maximum intensity of the device $(2.5 \mathrm{~T})$. Every patient received an explanation of the procedure and what to expect from it, and they were informed that the intense sensation was harmless. We also made sure that the temperature was comfortable for the patient.

Spastic muscles were stimulated for $1 \mathrm{~min}$ with an amplitude set at the motor threshold, and with a fixed frequency of $1 \mathrm{~Hz}$.

The antagonist muscles were facilitated during a $15 \mathrm{~s}$ long burst section, with a constant amplitude and constant frequency of $150 \mathrm{~Hz}$, then gradually strengthened during a $5 \mathrm{~min}$ long section that used a trapezoidal amplitude modulation with $2 \mathrm{~s}$ of amplitude increase until $100 \%, 1 \mathrm{~s}$ of plateau, $2 \mathrm{~s}$ of decrease and a $15 \mathrm{~s}$ pause, and a fixed frequency of $45 \mathrm{~Hz}$ (Table 1).

Table 1. Therapy parameters.

\begin{tabular}{ccccccc}
\hline & \multicolumn{6}{c}{ Sections } \\
& $\mathbf{1}$ & $\mathbf{2}$ & $\mathbf{3}$ & $\mathbf{4}$ & $\mathbf{5}$ & $\mathbf{6}$ \\
\hline Duration (sec) & 60 & 30 & 15 & 45 & 300 & 90 \\
Amplitude & Fixed & Fixed & Fixed & Fixed & $0-100 \%$ & Fixed \\
Frequency $(\mathrm{Hz})$ & 1 & 25 or 150 & 150 & 1 & 45 & 1 \\
\hline
\end{tabular}

\subsection{Patient Evaluation}

The patients were evaluated at the beginning of the treatment, at the completion of the therapy period (the 10th day of therapy), and on the 30th day from the completion of the therapy, by using the upper extremity functional index-Modified Ashworth Scale (MAS) - 
and the measurement of activities of daily living (ADL)-Barthel Score. All 60 patients completed the study by being present at the evaluation on the 30th day.

\subsection{Statistical Analysis}

In the case of qualitative variables, they were presented as absolute and relative frequencies. In the case of continuous, normally undistributed variables, or of the ordinal qualitative variables with more than three categories, they were presented with median (25th percentile-75th percentile). Comparisons between independent groups were made using the Chi-square or Fisher test when comparing frequencies, and with the Mann-Whitney test for continuous variables without normal distribution or ordinal quality variables. Normal distribution was tested with the Shapiro-Wilk test. Comparisons between dependent groups were made using the Wilcoxon signed-rank test for ordinal qualitative variables. SPSS 25.0 application was used for statistical analysis. The statistical significance threshold for the $p$-value was set at $p<0.005$.

\section{Results}

In the study group, there were $14(48.3 \%)$ men and 15 (51.7\%) women, while in the control group there were 16 (51.6\%) men and 15 (48.4\%) women. Both groups had an average age of 62 . There were no significant differences regarding sex $(p=0.796)$ and age $(p=0.722)$ between the two groups (Table 2).

Table 2. Groups comparison.

\begin{tabular}{cccc}
\hline Parameters & Study Group $(\boldsymbol{n}=\mathbf{2 9 )}$ & Control Group $(\boldsymbol{n = 3 1 )}$ & $p$ \\
\hline Male, no. $(\%)$ & $14(48.3)$ & $16(51.6)$ & $0.796^{* *}$ \\
Female, no (\%) & $15(51,7)$ & $15(48,4)$ & $0.7222^{*}$ \\
Age (years old) & $62(55 ; 71)$ & $62(57.5 ; 67.5)$ & \\
\hline Median (percentile 25th-75th), ${ }^{*} p$ obtained with Mann-Whitney test, ${ }^{* *} p$ obtained with Chi-square test.
\end{tabular}

In the study group, MAS presented a statistically significant decrease, both between the 1 st day and the 10 th day $(1.10 \pm 0.31, p<0.001)$, and between the 1 st day and the 30 th day $(0.83 \pm 0.47, p<0.001)$, and a significant increase between the 10th and 30th day $(-0.28 \pm 0.53, p=0.011)$. In the control group, MAS presented a statistically significant decrease between the 1 st day and the 10th day $(0.74 \pm 0.58, p<0.001)$, and between the 1 st day and the 30th day $(0.23 \pm 0.50, p=0.020)$, and a significant increase between the 10th and 30th day $(-0.52 \pm 0.51, p<0.001)$ (Figure 1$)$.

There was a significant increase in Barthel Score in both groups between the 1st and 10 th day of the study $(-2.28 \pm 1.58, p<0.001$ in the study group and $-2.42 \pm 1.48, p<0.001$ in the control group), and between the 1st and 30th day of the study $(-1.93 \pm 1.60, p<0.001$ in the study group and $-1.87 \pm 1.09, p<0.001$ in the control group), but we observed a decrease in Barthel Score in both groups between the 10th and 30th day $(0.35 \pm 0.94$, $p=0.064$ in the study group and $0.55 \pm 0.96, p=0.005$ in the control group) (Figure 2).

We also analysed the number of patients in each group that had an improved, unchanged, or worsened MAS or Barthel Score on the 10th and 30th day. There was a statistically significant difference between the study group and the control group regarding the percentage of participants with an improved MAS from the 1st day to the 10th day, with $32.3 \%$ more improved cases in the study group (100\% vs. 67.7\%, $p=0.004)$. When comparing MAS from day 1 with day 30, the study group had 59.9\% more improved cases than the control group $(79.3 \%$ vs. $19.4 \%, p<0.001)$. In the study group, in more than $50 \%$ $(68,97 \%)$ of participants, MAS values improved or remained unchanged until the 30th day, while in the control group, in more than 50\% (51.61\%) of cases, MAS values worsened, without statistical significance $(p=0.087)$. There were no other significant differences regarding the change of status between the moments of testing (Table 3). 


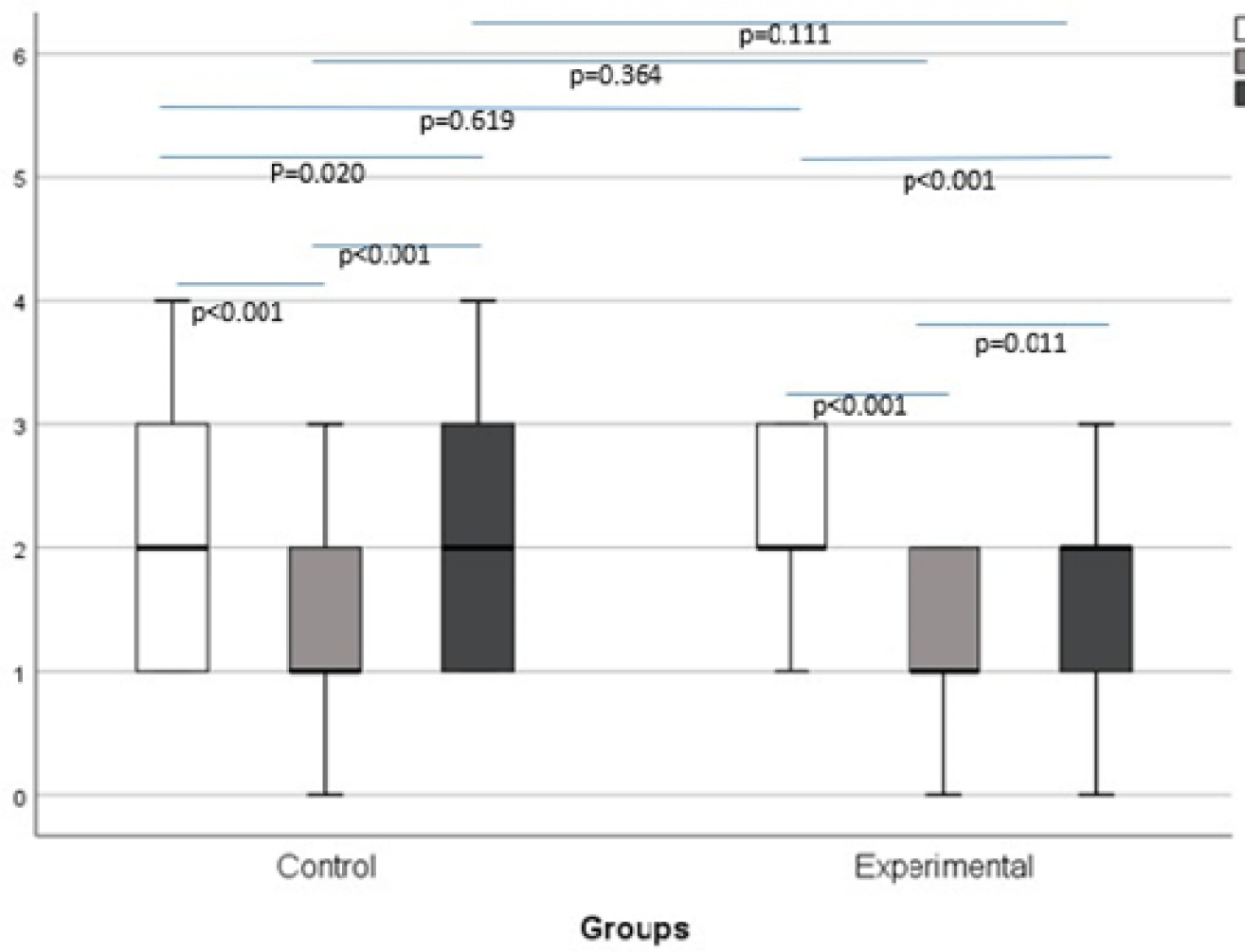

$\square$ MAS - initial (day 1)

MAS - day 10

MAS - day 30 post therapy

Figure 1. MAS mean comparison.

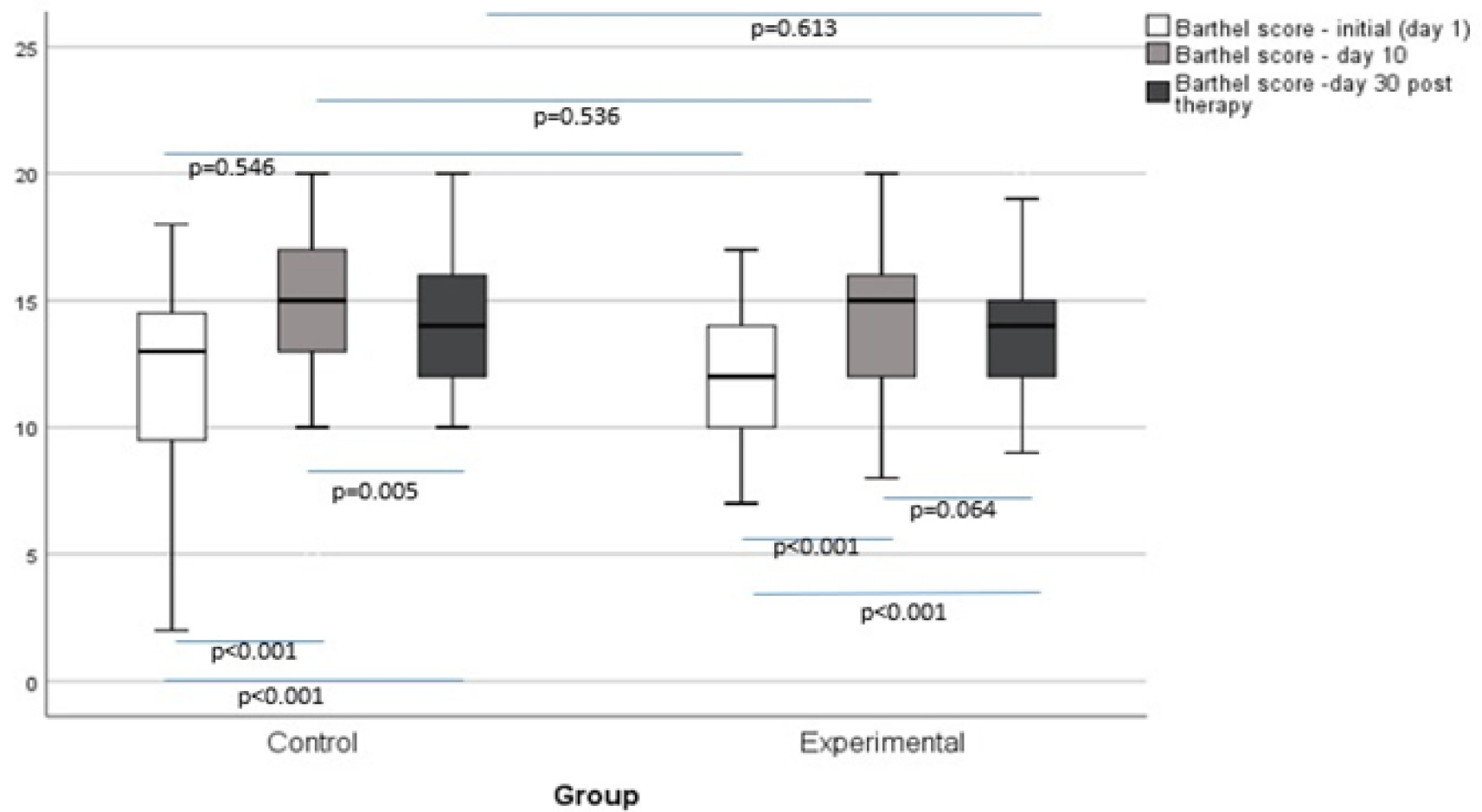

Figure 2. Barthel Score mean comparison. 
Table 3. Status analysis: improved, unchanged, or worsened between the moments of testing.

\begin{tabular}{|c|c|c|c|c|}
\hline & Status & Study Group $(n=29)$ & Control Group $(n=31)$ & $p$ \\
\hline \multirow{4}{*}{ Status MAS Day 1-Day 10, no (\%) } & improved & $29(100)$ & $21(67.74)$ & \multirow{3}{*}{0.004} \\
\hline & unchanged & $0(0)$ & $10(32.26)$ & \\
\hline & worsened & $0(0)$ & $0(0)$ & \\
\hline & improved & $23(79.31)$ & $6(19.35)$ & \multirow{4}{*}{$<0.001$} \\
\hline \multirow[t]{3}{*}{ Status MAS Day 1-Day 30, no (\%) } & unchanged & $6(20.69)$ & $25(80.65)$ & \\
\hline & worsened & $0(0)$ & $0(0)$ & \\
\hline & improved & $1(3.45)$ & $0(0)$ & \\
\hline \multirow[t]{3}{*}{ Status MAS Day 10-Day 30, no (\%) } & unchanged & $19(65.52)$ & $15(48.39)$ & \multirow{3}{*}{0.087} \\
\hline & worsened & $9(31.03)$ & $16(51.61)$ & \\
\hline & improved & $27(93.1)$ & $30(96.77)$ & \\
\hline \multirow[t]{3}{*}{ Status Barthel Day 1-Day 10, no (\%) } & unchanged & $2(6.9)$ & $1(3.23)$ & \multirow[t]{3}{*}{1} \\
\hline & worsened & $0(0)$ & $0(0)$ & \\
\hline & improved & $23(79.31)$ & $29(93.55)$ & \\
\hline \multirow[t]{3}{*}{ Status Barthel Day 1-Day 30, no (\%) } & unchanged & $5(17.24)$ & $2(6.45)$ & \multirow[t]{3}{*}{0.744} \\
\hline & worsened & $1(3.45)$ & $0(0)$ & \\
\hline & improved & $4(13.79)$ & $3(9.68)$ & \\
\hline \multirow[t]{2}{*}{ Status Barthel Day 10-Day 30, no (\%) } & unchanged & $13(44.83)$ & $14(45.16)$ & \multirow[t]{2}{*}{0.554} \\
\hline & worsened & $12(41.38)$ & $14(45.16)$ & \\
\hline
\end{tabular}

$p$ obtained with Mann-Whitney test.

\section{Discussion}

There was an improvement in MAS in both groups at the 10th and 30th day evaluations, in comparison with the evaluation performed on the first day, but when comparing the indexes from the last day of therapy (10th), with the indexes from the 30th day after treatment, we observed an increase in the values. Our results show that, within four weeks, the MAS values are not as low as they were on the last day of therapy.

The Barthel Scores improve on the last day of the therapy (10th day) and on the 30th day after treatment, in comparison with the first day of the therapy in both groups. However, it decreases significantly when comparing the value on the 10th day of therapy with the value on the 30th day after treatment in the control group, and without statistical significance in the study group, which means that the improvements in ADL are more persistent in the study group.

When comparing the two groups, the differences between MAS values between the 1st and the 10th day, and between the 1st and the 30th day, were more significant in the study group, which means that the 9 min protocol (with one extra minute of electromagnetic stimulation on the agonist muscles) had a better outcome. However, the differences in MAS values between the 10th and the 30th day did not achieve statistical significance when comparing the two groups, which means that the effects on spasticity of both protocols are attenuated in a 4 weeks' time in the same manner. Moreover, the percent of improved cases (from day 1 to day 10 of treatment, and from day 1 to day 30 after the completion of therapy) was statistically significantly higher in the study group, but there was no difference between groups when comparing the percentage of improved cases from the 10th day to the 30th day after the completion of therapy. Even if, in the study group, in more than $50 \%(68,97 \%)$ of participants, MAS values improved or remained unchanged until the 30th day, in the control group, in more than 50\% (51.61\%) of cases, MAS values worsened, and we did not achieve statistical significance $(p=0.087)$.

The reduction in MAS values 30 days after the discontinuation of treatment could be explained by the lack of continuity or neglect of adequate physical therapy at home by patients and/or the lack of daily monitoring of patients with decreased motivation and compliance with treatment. Patients and their families and caregivers should be adequately trained in physical therapy at home, as well as in the possibility of using virtual therapy and telerehabilitation. Telerehabilitation allows long-term treatment to be provided to 
patients in the current context of the COVID-19 pandemic, which greatly limits human movement and interaction [15].

There were no statistically significant differences regarding Barthel Score between the two groups in different moments of testing.

Significant changes in MAS values between groups (between day 1 of treatment and day 10 of treatment, and between day 1 of treatment and day 30 of post-treatment), compared with statistically insignificant changes in the Barthel Score between the two groups, may suggest that the results on the increase in the functionality and performance of ADLs are weaker than those obtained in reducing upper-limb spasticity in stroke patients.

Patients in both groups benefited from high-intensity electromagnetic stimulation (Super Inductive System), the difference between the groups being represented by the extra minute of agonist muscle therapy in the case of the study group. The action on the agonist muscles for one minute, in addition to the therapy on the antagonist muscles for $8 \mathrm{~min}$, does not bring additional benefits in terms of upper-limb function and the recovery of ADLs. A possible cause of these results may be the insufficient time of action of the therapy at the level of the agonist, spastic muscle group.

High-intensity electromagnetic therapy (Super Inductive System) has been shown to be effective in relieving pain, increasing mobility, and improving the quality of life of patients with acute and chronic musculoskeletal and neurological disorders in other studies [12].

In patients presenting spasticity after stroke, a study published in 2018 demonstrated the effectiveness of the Super Inductive System in reducing spastic muscle tone and in improving patient function while maintaining results, and even escalating them in a favourable sense of patient evolution, 1 month after completion of the treatment. In this study, the therapy was first applied to the agonist muscles for post-facilitatory inhibition, and later to the weak antagonistic muscles, in order to increase the tone of these muscles, a methodology similar to our study [9].

\subsection{Advantages}

Super Inductive System Therapy offers the advantage of the non-contact technique and the possibility of adjusting the intensity according to the patient's tolerance. The therapy also has a relatively small number of contraindications and few side effects, and can be applied to patients with very different pathologies.

\subsection{Limitations}

One limitation of our study that influenced the statistical significance of the results is the relatively small number of participants. Another limitation of our study is that we did not analyse the effect of the high-intensity electromagnetic stimulation according to the elapsed time from the acute stroke. It is considered that the neuroplasticity has the best treatment response in the first 6 months after stroke, but there is evidence that also suggests important outcomes of rehabilitation therapy in the chronic phase of a stroke [16,17].

On the other hand, the proposed time for stimulating the agonist muscles was probably insufficient, the results obtained on the functional improvement of the patients being without statistical significance.

Last but not least, it should be noted that the study was not double-blind, the evaluator knowing the group that the patient was part of in the evaluation at 10 days of treatment and 30 days after therapy.

\section{Conclusions}

High-intensity electromagnetic therapy improves spasticity and upper limb function in patients with post-stroke spasticity.

Applying therapy to agonist-antagonist muscles by restoring muscle balance brings benefits in reducing the tone of spastic muscles, but without significantly influencing the functionality of the upper limb in the long term. 
In order to obtain conclusions with an impact on the therapeutic management of poststroke spastic patients, extensive, multicentred, double-blind controlled studies are needed, as well as comparative studies of treatment protocols with standardized methodology.

Author Contributions: Conceptualization, V.M.C., I.M.B. and I.M.; methodology, all.; investigation, I.L., E.B.; resources, all; writing — original draft preparation, I.M., A.D.C.; writing—-review and editing, V.M.C., R.A.U., I.L., M.G.I.; visualization, I.M.B., I.M., C.I.B.; supervision, V.M.C., L.I., M.G.I., V.M.C., I.M., I.M.B., R.A.U., M.G.I. and L.I. had equal contributions. All authors have read and agreed to the published version of the manuscript.

Funding: This research received no external funding.

Institutional Review Board Statement: The study was conducted in accordance with the Declaration of Helsinki and approved by the Ethics Committee of "Iuliu Hatieganu" University of Medicine and Pharmacy (258/30 July 2019).

Informed Consent Statement: Informed consent was obtained from all subjects involved in the study.

Data Availability Statement: The data presented in this study are available on valid request from the corresponding authors. The data are not publicly available due to privacy and ethical concerns.

Conflicts of Interest: The authors declare no conflict of interest.

\section{References}

1. Donkor, E.S. Stroke in the 21st Century: A Snapshot of the Burden, Epidemiology, and Quality of Life. Stroke Res. Treat. 2018, 2018, 3238165. [CrossRef] [PubMed]

2. Li, L.; Scott, C.A.; Rothwell, P.M. Trends in Stroke Incidence in High-Income Countries in the 21st Century. Stroke 2020, 51, 1372-1380. [CrossRef] [PubMed]

3. Xu, H.; Wang, E.; Chen, F.; Xiao, J.; Wang, M. Neuroprotective Phytochemicals in Experimental Ischemic Stroke: Mechanisms and Potential Clinical Applications. Oxid. Med. Cell Longev. 2021, 2021, 6687386. [CrossRef] [PubMed]

4. Sakai, K.; Yasufuku, Y.; Kamo, T.; Ota, E.; Momosaki, R. Repetitive Peripheral Magnetic Stimulation for Impairment and Disability in People after Stroke. Cochrane Database Syst. Rev. 2019, 11, CD011968. [CrossRef] [PubMed]

5. Ganesh, A.; Luengo-Fernandez, R.; Rothwell, P.M. Late Functional Improvement and 5-Year Poststroke Outcomes: A PopulationBased Cohort Study. J. Neurol. Neurosurg. Psychiatry 2020, 91, 831-839. [CrossRef] [PubMed]

6. Rossini, P.M.; Burke, D.; Chen, R.; Cohen, L.G.; Daskalakis, Z.; Di Iorio, R.; Di Lazzaro, V.; Ferreri, F.; Fitzgerald, P.B.; George, M.S.; et al. Non-Invasive Electrical and Magnetic Stimulation of the Brain, Spinal Cord, Roots and Peripheral Nerves: Basic Principles and Procedures for Routine Clinical and Research Application. An Updated Report from an I.F.C.N. Committee. Clin. Neurophysiol. 2015, 126, 1071-1107. [CrossRef] [PubMed]

7. Huang, P.; Xu, L.; Xie, Y. Biomedical Applications of Electromagnetic Detection: A Brief Review. Biosensors 2021, 11, 225. [CrossRef] [PubMed]

8. Momosaki, R.; Yamada, N.; Ota, E.; Abo, M. Repetitive Peripheral Magnetic Stimulation for Activities of Daily Living and Functional Ability in People after Stroke. Cochrane Database Syst. Rev. 2017, 6, CD011968. [CrossRef] [PubMed]

9. Prouza, O.; Kouloulas, E.; Zarkovic, D. High-intesity electromagnetic stimulation can reduce spasticity in post-stroke patients. Int. J. Physiother. 2018, 5, 87-91. [CrossRef]

10. Kouloulas, E.J. Peripheral application of repetitive pulse magnetic stimulation on joint contracture for mobility restoration: Controlled randomized study. Int. J. Physiother. 2016, 3, 569-574. [CrossRef]

11. Krewer, C.; Hartl, S.; Müller, F.; Koenig, E. Effects of Repetitive Peripheral Magnetic Stimulation on Upper-Limb Spasticity and Impairment in Patients with Spastic Hemiparesis: A Randomized, Double-Blind, Sham-Controlled Study. Arch. Phys. Med. Rehabil. 2014, 95, 1039-1047. [CrossRef] [PubMed]

12. Zarkovic, D.; Kazalakova, K. Repetitive peripheral magnetic stimulation as pain management solution in musculoskeletal and neurological disorders: A pilot study. Int. J. Physiother. 2016, 3, 721-725. [CrossRef]

13. Irsay, L.; Checicheș, A.; Perja, D.; Borda, I.M.; Dogaru, G.; Onac, I.; Ungur, R.; Ciortea, V. Pharmacological Pain Management in Patients with Chronic Kidney Disease. Balneo 2019, 10, 12-16. [CrossRef]

14. Irsay, L.; Checiches, A.; Perja, D.; Borda, I.M.; Dogaru, G.; Ungur, R.; Ciubean, A.; Ciortea, V. Pharmacological Pain Management in Patients with Chronic Hepatic Disease. Balneo 2019, 10, 119-123. [CrossRef]

15. Ciortea, V.M.; Motoașcă, I.; Ungur, R.A.; Borda, I.M.; Ciubean, A.D.; Irsay, L. Telerehabilitation—A Viable Option for the Recovery of Post-Stroke Patients. Appl. Sci. 2021, 11, 10116. [CrossRef] 
16. Rozevink, S.G.; van der Sluis, C.K.; Garzo, A.; Keller, T.; Hijmans, J.M. HoMEcare ARm RehabiLItatioN (MERLIN): Telerehabilitation Using an Unactuated Device Based on Serious Games Improves the Upper Limb Function in Chronic Stroke. J. NeuroEng. Rehabil. 2021, 18, 48. [CrossRef] [PubMed]

17. Platz, T. Clinical Pathways in Stroke Rehabilitation: Evidence-Based Clinical Practice Recommendations; Platz, T., Ed.; Springer International Publishing: Cham, Switzerland, 2021; ISBN 978-3-030-58504-4. 